\title{
Mappemonde
}

Revue trimestrielle sur l'image géographique et les formes du territoire

\section{Données institutionnelles et données contributives sur la biodiversité, quelle légitimité ?}

Levels of legitimacy of institutional and contributive data on biodiversity, what legitimacy?

La legitimidad de la información institucional o colaborativa sobre la

biodiversidad

Xavier Amelot, Laurent Couderchet et Matthieu Noucher

\section{(2) OpenEdition}

Journals

Édition électronique

URL : http://journals.openedition.org/mappemonde/2861

ISSN : 1769-7298

\section{Éditeur}

UMR ESPACE

\section{Référence électronique}

Xavier Amelot, Laurent Couderchet et Matthieu Noucher, « Données institutionnelles et données

contributives sur la biodiversité, quelle légitimité ? », Mappemonde [En ligne], 120 | 2017, mis en ligne le 01 avril 2017, consulté le 12 juin 2020. URL : http://journals.openedition.org/mappemonde/2861

Ce document a été généré automatiquement le 12 juin 2020.

\section{(c) (i) (3)}

La revue Mappemonde est mise à disposition selon les termes de la Licence Creative Commons Attribution - Pas d'Utilisation Commerciale - Partage dans les Mêmes Conditions 4.0 International. 


\section{Données institutionnelles et données contributives sur la biodiversité, quelle légitimité ?}

Levels of legitimacy of institutional and contributive data on biodiversity, what legitimacy?

La legitimidad de la información institucional o colaborativa sobre la biodiversidad

Xavier Amelot, Laurent Couderchet et Matthieu Noucher

\section{Introduction}

Les politiques de protection des habitats, de la flore et de la faune, réunies sous le terme générique de politiques de conservation de la biodiversité, sont progressivement devenues grandes consommatrices d'information géographique. Depuis les années 1990, avec la mise en œuvre du réseau Natura 2000 jusqu'à aujourd'hui pour la réalisation des Trames vertes et bleues (TVB) du Grenelle de l'environnement, cette information est devenue indispensable à la mise en œuvre de l'action environnementale en faveur d'une biodiversité désormais prise en compte sur l'ensemble des territoires: biodiversité ordinaire, continuités écologiques. Dans ce contexte de demande croissante en information géographique sur la biodiversité, on observe un double mouvement: d'une part, la faiblesse des moyens consacrés par les pouvoirs publics à la reconnaissance des milieux selon ce qui pourrait être considéré comme "les règles de l'art»; d'autre part, la montée en puissance de démarches initiées hors de la sphère institutionnelle. Ces dernières diffusent des productions aux appellations variées : données « d'amateurs ", «volontaires » ou " citoyennes », que M. Goodchild (2007) a réunies sous l'expression « volunteered geographic information » $\left(\mathrm{VGI}^{1}\right)$ dans ses travaux sur les citoyens-capteurs. 
2 Les territoires sont à la fois une construction sociale et une matérialité signalait l'appel à communication du colloque 2014 du Collège International des Sciences du Territoire $(\mathrm{CIST})^{2}$ dont est issu ce dossier thématique de M@ppemonde. De ces deux volets d'une même réalité, les sciences de la nature privilégient la matérialité lorsqu'elles proposent leur recours vis-à-vis des politiques de conservation. La nature est alors précisément décrite, selon des protocoles scientifiquement validés, mais sur les espaces réduits faisant l'objet des zonages de protection. Pour le reste, les pouvoirs publics récupèrent «à la volée » des données existantes sur des lieux, des sites ou des territoires ; données qui peuvent, ou non, passer au crible de structures de validation, le Museum National d'Histoire Naturelle (MNHN) notamment, avant d'intégrer des bases d'informations plus institutionnelles. C'est ainsi que s'est structuré le fichier ZnieFF ${ }^{3}$, véritable fondement de la connaissance officielle de la biodiversité en France (Couderchet et Amelot, 2010).

3 Des organisations non gouvernementales constituent et diffusent désormais de leur côté des bases de données indépendantes. Les atlas de faune interactifs mis en ligne par la Ligue de Protection des Oiseaux (LPO) fournissent ainsi une nouvelle représentation de la nature, à l'image, pour la région Aquitaine, du dispositif Faune-Aquitaine: https://www.faune-aquitaine.org, à la fois complémentaire et concurrent des données officielles. Ils permettent aussi aux utilisateurs de ce type de plateforme de contribuer à l'enrichissement de l'information en apportant leurs propres observations.

Les cloisons entre la sphère de l'institutionnel et celle du volontaire ne sont cependant pas étanches ; les collectivités en manque de connaissances sur leurs propres territoires mettent en place des plateformes du même type. Bordeaux Métropole construit son atlas contributif de la biodiversité avec l'aide d'associations locales, dont la LPO (http:// www.bordeaux-metropole.fr/projets-nature/atlas-de-la-biodiversite); le MNHN lui-même, garant de la légitimité des données officielles, porte le programme VigieNature (http://vigienature.mnhn.fr). Ce programme, initialement focalisé sur les oiseaux avec, depuis 1989, le "Suivi Temporel des Oiseaux Communs» (STOC), concerne aujourd'hui une grande variété de groupes (papillons, chauves-souris, escargots, insectes pollinisateurs, libellules, plantes sauvages des villes) notamment à travers l'observatoire contributif de la biodiversité des jardins (http://obj.mnhn.fr).

Cette profusion d'informations n'est pas unanimement appréciée par les scientifiques et les organismes officiels. Sa légitimité est même parfois remise en cause (AFIS, 2013 ; 2015). Un rapport d'autorité s'instaure entre la donnée de la sphère scientifique, robuste, mais confidentielle; la donnée technique, produite localement à la demande des pouvoirs publics par des bureaux spécialisés et qui adopte rapidement un caractère opposable ; la donnée volontaire, produite par la foule ${ }^{4}$ et largement diffusée. Cette dernière famille de données compense son caractère non institutionnel par les formes ouvertes de sa production et délibératives de sa validation. L'information sur la biodiversité s'inscrit ainsi dans un système de valeurs démocratiques qui oppose les dispositifs technocratiques aux dispositifs participatifs. Il n'y a pourtant pas d'un côté des données officielles, fiables, et de l'autre des données d'amateurs avec tout ce que le terme peut avoir de péjoratif. La légitimité des données, qu'elles soient « amateurs » ou « institutionnelles », nous semble devoir être dans tous les cas, questionnée d'abord du point de vue de leurs spécificités intrinsèques :

- la nature des données et des objets qu'elles représentent (habitats vs espèces, observations vs analyses) ; 
- la régularité spatiale et temporelle de la couverture (exhaustivité, résolution, échelle, mise à jour); la structuration de l'information selon des normes précises, dans les «règles de

l'art »;

- la souveraineté précisant les modalités de collecte, la propriété ou encore les règles de diffusion.

Mais, dans le contexte actuel d'enchevêtrement des parties prenantes dans la production de données, la seule prise en compte de ces spécificités intrinsèques, autrement dit des métadonnées, ne peut être sufFisante. Une approche permettant de combiner l'étude de la qualité (interne et externe) et de la crédibilité des données géographiques peut alors permettre de questionner leur légitimité. La qualité interne se mesure au travers de critères (précision géométrique, exhaustivité, précision sémantique, cohérence logique, actualités, etc. $)^{5}$ qui permettent d'évaluer l'adéquation entre les spécificités techniques et la réalisation efFective ; alors que la qualité externe se définit plutôt comme une adéquation aux besoins ${ }^{6}$ (Devillers et Jeansoulin, 2005). La crédibilité renvoie à la fois au contexte de production et au degré d'ambiguïté de la donnée : plus le contexte de production est simple et plus l'ambiguïté autour de la source et des conditions d'acquisition sont faibles, plus la donnée peut être considérée comme crédible (Flanagin et Metzger, 2008). Dès lors, les approches centrées sur les spécifications techniques des données nous semblent devoir être complétées par une analyse plus large du contexte de leur fabrique (Joliveau et al., 2013) et de leurs usages (Amelot, 2013). Ainsi, proposons-nous d'analyser l'inventaire des ZniefF et l'atlas de faune d'Aquitaine comme des dispositifs sociotechniques. Cette notion intègre les caractéristiques techniques dans une matrice plus large aux contours flous qui s'individualise sous l'efFet de stratégies et d'ajustements multiples et parfois contradictoires. Elle nous permet d'insister sur la dynamique des rapports qui s'instaurent au sein des fabriques de données de la biodiversité et sur les valeurs et visions de la régulation politique que véhiculent leurs usages (ou leur non-usage) : « Les choix d'instruments sont significatifs des choix de politiques publiques et des caractéristiques de ces dernières. On peut alors les envisager comme des traceurs, des analyseurs des changements. Le type d'instrument retenu, les propriétés de celui-ci et les justifications de ces choix nous semblent souvent plus révélateurs que les exposés des motifs et les rationalisations discursives ultérieures » (Lascoumes et Le Galès, 2005, p. 28). Il s'agit alors, en s'inscrivant dans la continuité des travaux sur la cartographie critique (Robinson, 1952; Harley, 1988; Pickles 2004; Dodge et Kitchin, 2007), de contribuer à interroger ces dispositifs sociotechniques et, ce faisant, à repenser l'ensemble des données de la biodiversité comme des formes de savoir socialement construit, subjectif et idéologique (Lascoumes, 2007).

\section{Les Znieff, un inventaire robuste?}

7 La base de données ZniefF constitue un des piliers des politiques de conservation de la nature en France. Nous avons proposé l'idée que cette base de connaissances, bien qu'elle ne s'en revendique pas, soit considérée comme participative (Amelot et Couderchet, 2012), bien avant qu'apparaisse le développement de la cartographie 2.0 (Mericskay et Roche, 2011). Le fichier ZniefF est en efFet une compilation et une mise en cartes de connaissances naturalistes construites à partir de l'échelon local, notamment par les observations de terrain des membres de sociétés savantes. Ces 
connaissances collectées par l'administration régionale de l'environnement font l'objet d'une première validation, à l'échelle régionale, par les Conseils Scientifiques Régionaux de Protection de la Nature (CSRPN), assemblées constituées intuitu personae parmi «les notabilités des sciences $»^{7}$. Le Museum National d'Histoire Naturelle (MNHN) en opère une seconde au niveau national et se charge de l'intégration de ces connaissances dans une base nationale de données géographiques qui pourra être ensuite diffusée à l'échelon national (par le MNHN) et à l'échelon régional par les services déconcentrés du ministère responsable de l'écologie (Directions Régionales de l'Environnement, de l'Aménagement et du Logement, DREAL).

8 Lancée en 1982, la première génération du zonage ZniefF recensait plus de 14000 zones et concernait $17 \%$ du territoire métropolitain. Un second «inventaire » a été engagé entre 1995 et 2012 et se poursuit actuellement, «il couvre plus de $25 \%$ du territoire, recense plus de 20000 espèces et affiche plus d'un million de données!

9 Encore aujourd'hui, l'inventaire ZniefF n'a pas d'équivalent en Europe» (http:// znieff-2012.mnhn.fr).

10 L'administration et le MNHN présentent la base ZniefF comme un inventaire régulier et continu de la biodiversité (http://inpn.mnhn.fr/programme/inventaire-zniefF/ presentation) comme l'illustre la figure $\mathbf{1}$. $\mathrm{Si}$, sur certaines plateformes de visualisation et de diffusion des données, la coexistence de deux générations de fichiers ZniefF est explicite, elle ne l'est pas systématiquement comme sur la carte de la figure 1 (ou sur le Géoportail IGN). Cette carte, très homogène traduit la volonté de normalisation des données de la part des institutions. En effet, après les expérimentations de la production du premier fichier national, les données de la seconde génération de la base passent désormais au crible de bordereaux standard imposés par le MNHN pour en renforcer l'homogénéité. Peine perdue ? Les contrastes entre des régions couvertes de zonages et d'autres beaucoup plus faiblement concernées demeurent et les discontinuités locales des zonages au niveau des limites administratives se perpétuent ( figures 2 et 3). Le point de vue officiel justifie en partie ces contrastes par les spécificités écologiques régionales, les régions du sud-est du pays étant beaucoup plus riches en biodiversité que celles de la façade ouest (Siblet, 2012) et l'académique vient en soutien: «La qualité du processus de réalisation confère à l'inventaire une plus grande fiabilité » (Mathevet et al., 2013).

11 L'examen critique de la carte des ZniefF montre pourtant des faits troublants. Ainsi, la frontière entre les régions Auvergne et Limousin coupe le Massif Central en deux, comme si le fait de passer en région Limousin réduisait la richesse en biodiversité du massif (figures 2 et 3). De la même manière, la délimitation des ZniefF bute toujours localement sur les limites administratives régionales. La continuité écologique ne semble pas assurée pour certains types de milieu tel le bocage, par exemple retenu en région Pays de la Loire dans l'est de la Vendée, mais sans que ses prolongements bressuirais et gâtinais n'aient été pris en compte dans la région voisine du PoitouCharentes (figure 3 repère a). Ces discontinuités locales sont parfois liées aux discordances de génération du fichier comme dans l'est de la région Aquitaine aux confins de la Dordogne et du Lot (figure 3 repère b). Cependant, même pour des zonages relevant de la seconde génération les discontinuités persistent comme pour la région Poitou-Charentes dont la ZniefF forestière du sud bute sur les limites de la Gironde en Aquitaine (figure 3 repère c). Les contrastes régionaux marqués que nous avions mis en évidence dans la base de première génération (Couderchet et Amelot, 
2010) semblent donc bien persister dans la seconde. À défaut de constituer un véritable inventaire de la biodiversité à l'échelle nationale, la base ZniefF demeure un remarquable inventaire des connaissances sur la biodiversité, cette connaissance étant beaucoup plus développée pour certaines régions et pour certains groupes d'espèces que pour d'autres.

12 Même s'il est relayé par l'institution, le fichier ZniefF est donc fondamentalement une base de connaissances et non un inventaire, homogène, régulier et exhaustif. Il demeure le fruit de contributions d'amateurs et, en cela, il se rapproche des bases de données contributives qui se développent aujourd'hui et dont il partage la richesse, mais aussi les limites.

Figure 1. Capture d'écran du portail cartographique de l'Inventaire National du Patrimoine Naturel

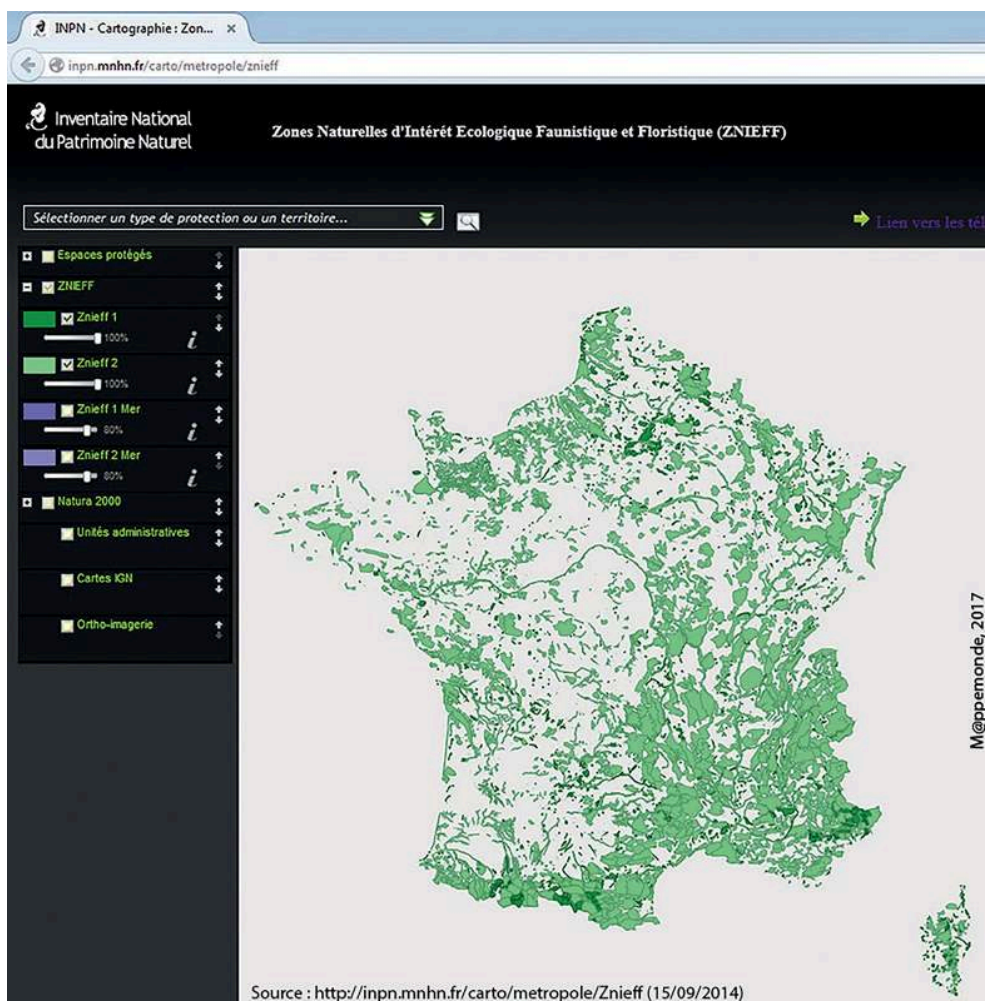

Source : http://inpn.mnhn.fr/carto/metropole/Znieff, consulté le 15/09/2014, remplacé depuis : https://inpn.mnhn.fr/viewer-carto/espaces 
Figure 2. Les contrastes régionaux de la base de données Znieff actualisée (au 15/09/2014)

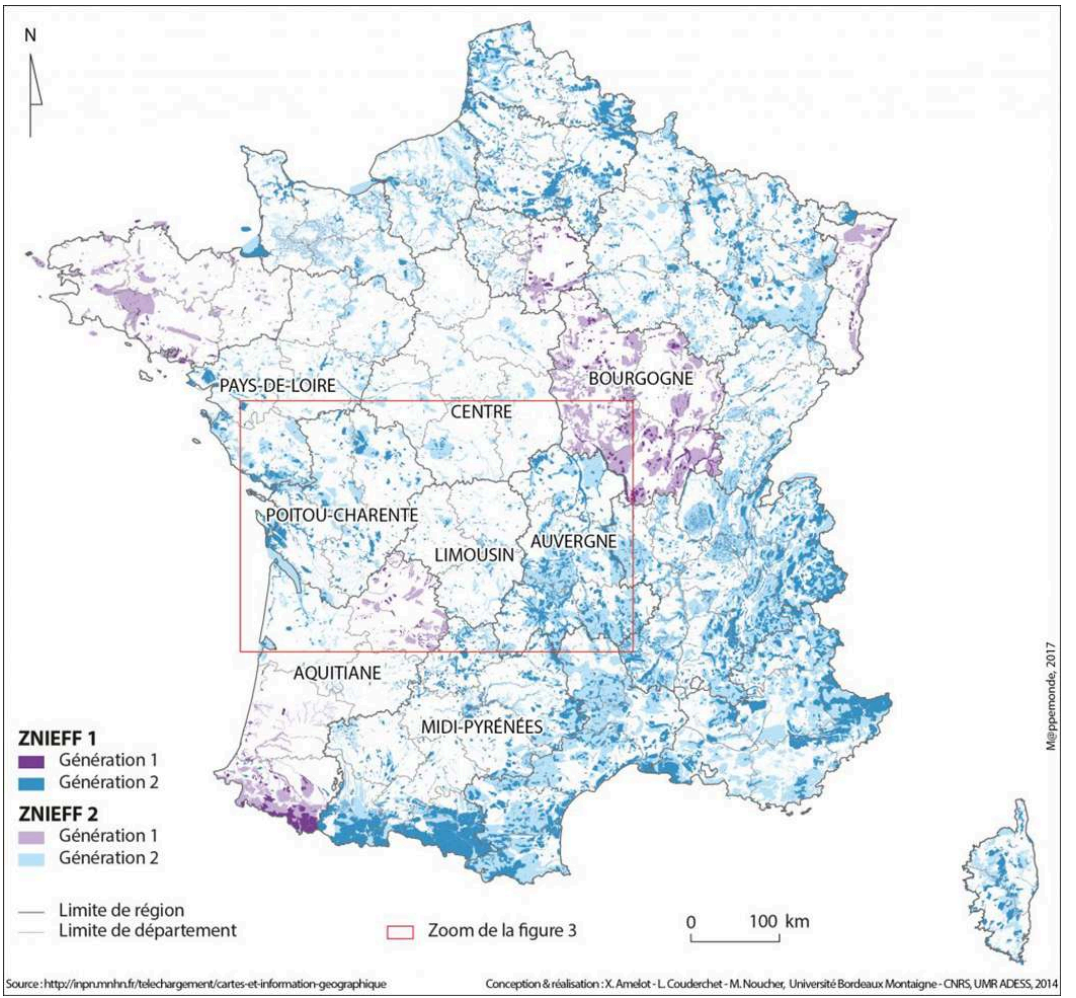

Carte réalisée à partir des couches d'information ZNIEFF 1 et ZNIEFF 2 téléchargeables sur le site http://inpn.mnhn.fr/telechargement/cartes-et-information-geographique, fichiers téléchargés le 15/09/2014 
Figure 3. Les contrastes régionaux de la base de données Znieff actualisée (au 15/09/2014)

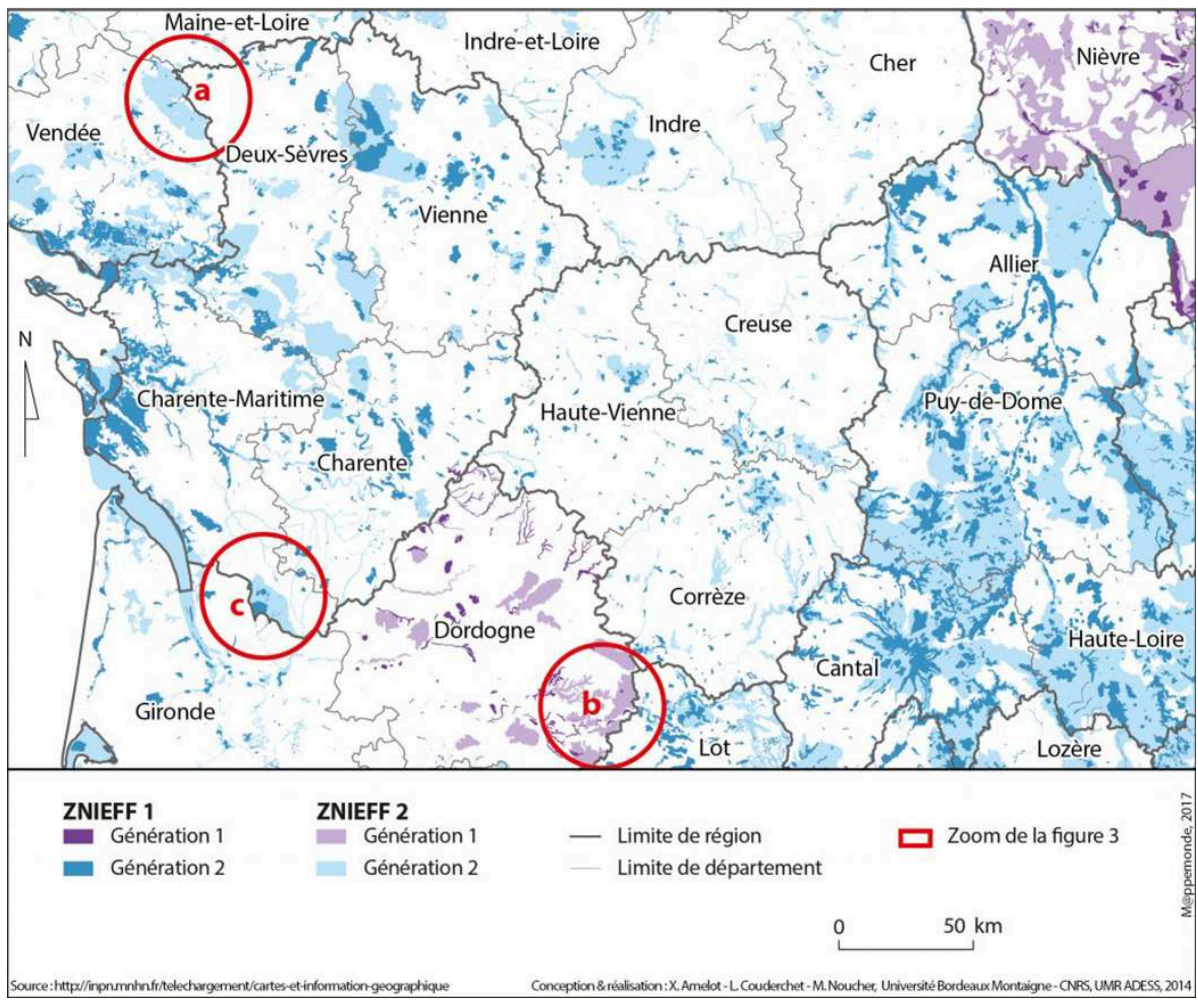

Carte réalisée à partir des couches d'information ZNIEFF 1 et ZNIEFF 2 téléchargeables sur le site https://inpn.mnhn.fr/telechargement/cartes-et-information-geographique, fichiers téléchargés le $15 / 09 / 2014$

\section{L'expérience de l'atlas de faune d'Aquitaine}

Réunies initialement sous la forme de collections d'inventaires au format papier, les données de faune connaissent un processus progressif de rationalisation. L'une des facettes essentielles de cette rationalisation a consisté à intégrer une dimension géographique à ces données. À partir de la fin des années 1990, ces données géographiques commencent lentement à être diffusées sur Internet et désormais, avec les technologies du web 2.0, c'est même la saisie et le contrôle en ligne qui sont possibles. La double transition numérique (dématérialisation des carnets d'observation et possibilité de diffusion massive des données) que connaît le monde naturaliste n'est pas sans conséquence sur le renouvellement des pratiques d'observation comme sur les possibilités nouvelles d'analyse, avec l'émergence de nouveaux contributeurs et de nouveaux usages (Noucher et Gautreau, 2012).

14 À titre d'exemple, pour la seule région Aquitaine, la plateforme Faune Aquitaine (https://www.faune-aquitaine.org/) comptait, en août 2013, plus de 1800 contributeurs actifs et près de 2,3 millions d'enregistrements (Liger et al., 2013), soit plus de deux fois plus que la base ZniefF pour la France entière. Comme pour les ZniefF, les données publiées sur le site sont validées par un organe de contrôle d'une cinquantaine de spécialistes des différents secteurs du règne animal. Toute donnée peut ainsi être discutée au sein du comité de validation et avec son auteur, sa validité est négociée. Pendant ce temps de négociation, la donnée figure sur le site, mais une icône «?» 
symbolise le fait qu'elle est sous la responsabilité de son auteur, et qu'elle n'est pas incluse dans les synthèses. L'icône "? " agit ainsi comme une forme d'alerte. S'engage alors une négociation qui repose notamment sur un forum interne où les membres peuvent interpeler le contributeur pour lui demander des précisions (description ou comportement de l'animal, photo, conditions édaphiques) et comparer avec l'historique de la base afin de pouvoir "trianguler " l'observation avec d'autres éléments. L'enjeu est alors l'établissement d'un consensus permettant à la contribution d'acquérir une légitimité publique suffisante pour intégrer définitivement et sans réserve la base de connaissances. Ces disputes d'ordre épistémique, qui concernent la légitimité ou la justesse d'une connaissance participent au renforcement de la dynamique collective, à la mise en commun de savoir-faire et, in fine, à la constitution d'une communauté de pratique (Lave et Wenger, 1991). Le dispositif est contributif, mais il est précisément encadré, les données jugées non crédibles à l'issue des discussions sont maintenues avec l'icône «? » ou supprimées par leur auteur.

La LPO semble assumer, davantage que les pouvoirs publics pour les ZniefF, les caractéristiques inhérentes au procédé de collecte de ses données et les limites d'utilisation de la cartographie qui en résulte. La base de données pointe des lieux où les animaux ont été vus, elle ne prétend pas qu'ils ne soient pas présents là où leur observation n'a pas été portée sur le site. Une collaboration entre la LPO et notre équipe de recherche (UMR Adess CNRS, Universités de Bordeaux) est engagée depuis 2013 pour mesurer, à partir d'une analyse du contenu de la base, les efFets de la méthode sur la qualité du produit. Elle montre précisément le lien entre les terrains de prospection des contributeurs et la densité d'observation dans l'espace, établissant même une relation sans équivoque entre l'adresse du domicile de l'observateur et la pression d'observation (Liger, 2013). Elle révèle aussi une forme de tropisme exercé par les aires protégées vers lesquelles se déplacent plus volontiers les observateurs que vers des espaces sans qualité remarquable (Razer, 2014). Une forme de circularité s'établit donc entre connaissance et protection: "c'est connu donc c'est protégé »; "c'est protégé donc c'est observé et c'est (re-)connu ». À l'inverse, des zones blanches d'observation sont mises en évidence : non connues donc non protégées, non protégées donc non observées et non connues.

La base vise la connaissance la plus exhaustive et représentative possible de la faune de la région, son procédé de fabrication présente néanmoins, sur ce registre, une contradiction: les contributeurs privilégiant l'espèce exceptionnelle à l'espèce ordinaire, la seconde est proportionnellement moins représentée dans le fichier. La crédibilité de la base est cependant considérablement renforcée par la masse d'informations et l'exigence de traçabilité de la donnée que se fixe l'opérateur. La figure de l'amateur tend aussi à être remise en cause dès lors qu'on analyse le profil des contributeurs. Une enquête réalisée en 2013 auprès des 1800 contributeurs actifs de Faune Aquitaine révélait ainsi que la moitié d'entre eux considère avoir un métier proche du milieu naturaliste. Ce type de dispositif apparait également pour de nombreux contributeurs comme un levier pour professionnaliser une passion. Dès lors, l'amateur est à définir ici dans le sens de " celui qui aime » plus que comme l'antonyme du professionnel, aux connotations négatives de dilettantisme et d'incompétence.

17 On comprend donc l'intérêt que lui portent certaines collectivités territoriales pour orienter leurs propres politiques de conservation: la métropole de Bordeaux et le Département de la Gironde se sont associés au dispositif Faune Aquitaine en ce sens. La 
porosité entre données institutionnelles et données privées, mise en évidence par le jeu du crowdsourcing, tend donc à renforcer l'idée de frontières organisationnelles de plus en plus floues voire mouvantes entre producteurs, utilisateurs et difFuseurs de données géographiques (Noucher et Gautreau, 2012). L'analyse de ce type d'initiative donne des clés de compréhension complémentaires de la connaissance de la nature en général qui aident à comprendre d'autres bases de données comme celle des ZniefF (figure 4).

Figure 4. Circulation de l'information dans le dispositif sociotechnique étudié

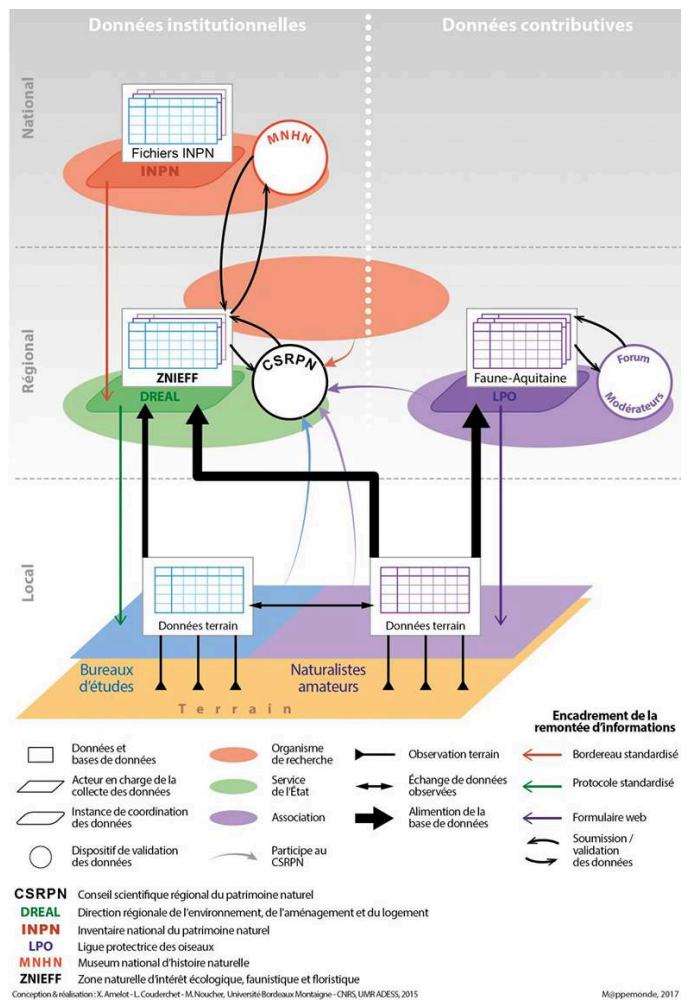

Conception et réalisation : X. Amelot - L. Couderchet - M. Noucher, Université Bordeaux Montaigne CNRS, UMR ADESS, 2015

Le schéma distingue les circuits de l'information institutionnelle et ceux de l'information volontaire, mais place le milieu associatif naturaliste comme source commune. Les naturalistes amateurs ont très largement contribué à la constitution du fichier ZniefF et participent désormais à la dynamique de la plateforme Faune Aquitaine. Cette réalité explique que l'on retrouve des caractéristiques communes aux deux bases. La seconde génération des ZniefF mobilise en partie des bureaux spécialisés dans l'observation de terrain qui travaillent à partir de bordereaux standard établis par l'instance centrale de validation. L'objectif de l'État est de renforcer l'homogénéité nationale de la base, mais nous observons qu'il n'est pas atteint (figures 2 et 3). La faiblesse du financement du programme ainsi qu'une réinterprétation militante locale des enjeux peuvent être avancés comme explication du phénomène. Mathevet et al. (2013) évoquent à ce propos une sollicitation des régions par l'État «avec des succès divers » qui justifient la mobilisation du milieu « associatif naturaliste dont on espère une participation largement bénévole ». Enfin, l'instance de validation est en partie le lieu où l'autorité scientifique institutionnelle est mobilisée. À travers le MNHN, bien sûr, au niveau national, mais aussi les CSRPN dans les régions, qui recrutent en partie à 
l'université. La légitimité scientifique est ainsi assurée. Une partie des membres du CSRPN est aussi issue du monde associatif, actif dans la production de données contributives, voire dans ses instances de validation. On comprend ainsi que la frontière entre les données issues de ces deux filières soit difficile à établir.

\section{De la connaissance de la nature à sa conservation}

La participation citoyenne est désormais constitutive des nouvelles politiques environnementales territorialisées. Elle pose la question de la capacité des connaissances citoyennes à animer le débat territorial. La production récente des Schémas Régionaux de Cohérence Écologique (SRCE) dans le cadre de la politique Trame Verte et Bleue du Grenelle de l'environnement, que la loi souhaite participative, montre une série d'ambiguïtés sur ce registre. Les ZniefF y sont considérées comme « réservoirs de biodiversité » ou des éléments devant être privilégiés dans l'établissement des trames sans que la relativité de leur fiabilité ne soit discutée (Allag-Dhuisme et al., 2010). Même si les textes issus du Grenelle privilégient la faune et ses mouvements dans une optique de préservation de corridors écologiques, les contributions du type de Faune Aquitaine n'interviennent pas dans les procédures. Cette sélection de la donnée considérée fiable aux dépens de celle qui le serait moins ne traduit-elle pas la crainte des acteurs traditionnels de la conservation de voir le pouvoir leur échapper avec l'arrivée de connaissances nouvelles?

Les données contributives ouvrent des pistes prometteuses pour la connaissance scientifique de la nature. L'atlas de faune identifie des lieux où ont été vus des animaux. Il dissocie le biotope de la biocénose, donne de la nature une conception non écosystémique. Il contrarie ce qui peut être considéré comme une doctrine de la conservation sur son volet scientifique, mais aussi politique. La Directive européenne Habitats de 1992 impose l'entrée par le biotope pour le «maintenir dans un bon état de conservation " de manière à préserver des espèces inféodées à ces habitats qui sont politiquement considérées « d'intérêt prioritaire ». L'étude de la relation de l'espèce à son habitat depuis sa position reconnue dans les atlas contributifs pourrait faire évoluer la connaissance de cette relation, les espèces étant observées dans des milieux différents dont certains ne correspondent pas à leur biotope : comment justifier l'entrée par le biotope? Ces données ne fourniraient-elles pas des éléments de connaissance sur la mobilité des espèces et les continuités écologiques?

Les interactions entre données contributives et zonages institutionnels sont parfois déjà engagées. Ainsi, deux missions de terrain organisées entre 2010 et 2014 nous ont permis de rencontrer les acteurs de la gouvernance informationnelle de la biodiversité en Guyane: services de l'État, collectivités territoriales, laboratoires de recherche, associations de protection de l'environnement, bureaux d'études (spécialisés dans le domaine naturaliste ou minier), instances de représentation des communautés amérindiennes et bushinengue. Les revendications corporatistes (demande d'un accès facilité aux ressources naturelles), environnementales (demande d'un encadrement strict des activités sur les espaces protégés) et autochtones (demande d'une meilleure prise en compte des droits d'usage) témoignent d'appropriations fortement différenciées de la question de la protection de la biodiversité (Oder, 2011). Elles ont cependant en commun de produire des données géographiques pour appuyer leurs revendications voire pour contrer certaines expertises (Noucher, 2017). Dans ce 
" combat de cartes", certaines alliances s'opèrent et une hybridation des sources institutionnelles et contributives peut alors s'observer. Ainsi, la Direction de l'Environnement de l'Aménagement et du Logement (DEAL) fait régulièrement appel au Groupe d'Étude et de Protection des Oiseaux de Guyane (GEPOG) pour expertiser ou contre-expertiser des diagnostics environnementaux à partir des données de fauneguyane.fr. Une telle démarche serait difficilement envisageable dans la plupart des régions métropolitaines, mais le contexte guyanais bouleverse ici la traditionnelle répartition des tâches et favorise les rapprochements entre l'État et les ONG. La richesse, mais aussi la connaissance très lacunaire de la biodiversité faunistique et floristique du territoire (MEDTL, 2010), les conflits récurrents autour de zonages visant à encadrer la protection de la biodiversité (Tsayem Demaze, 2008) ou l'aménagement des activités minières (Transler et al., 2006), la faiblesse des moyens de l'État que traduit la pauvreté des référentiels géographiques ${ }^{8}$, l'hyperconcentration des acteurs de la biodiversité sur Cayenne ${ }^{9}$ et les passerelles professionnelles dans le milieu naturaliste guyanais entre associations, bureaux d'étude et services déconcentrés de l'État ${ }^{10}$ sont autant de paramètres qui inscrivent les données de la biodiversité de Guyane dans un dispositif sociotechnique très spécifique... mais potentiellement précurseur. On peut en efFet s'interroger sur la généralisation, à terme, de l'hybridation de sources hétérogènes de données comme conséquence directe de la complexification des flux d'information géographique.

\section{Conclusion}

L'évolution des doctrines de l'action territoriale est marquée par des efFets d'inertie que traduit de façon assez spectaculaire la confrontation d'une base de données telle que la base Zniefr avec un atlas contributif tel que Faune Aquitaine. Si les évolutions technologiques ouvrent des possibilités de collecte d'informations considérables et démultiplient les possibilités de contribution à la production de connaissances pour l'aménagement du territoire, les principes de cette contribution préexistent au web 2.0. C'est ce que nous avons tenté de montrer avec l'exemple original de la base ZniefF. On observe aussi une série de formes intermédiaires entre des productions spontanées de données et des formes plus encadrées. Si la connaissance « OFFIcielle» est bordée par des dispositifs normatifs, la base contributive soumet, elle aussi, la donnée à une procédure de validation scientifique. On observe donc un degré plus ou moins élevé d'ouverture des bases aux observations citoyennes plutôt qu'une opposition entre des bases officielles et des bases citoyennes.

Nama Budhathoki (2008) traduit cette complexification par un schéma qui oppose deux modèles de flux (figure 5). Le modèle de "l'information géographique traditionnelle " est monodirectionnel : du producteur expert vers l'utilisateur expert voire, dans une moindre mesure, vers l'utilisateur amateur. Le modèle de «l'information géographique bénévole » est multidirectionnel : utilisateurs et producteurs, amateurs et experts sont en capacité de difFuser des flux d'information (figure 5). 
Figure 5. Évolution des modes de production/diffusion de l'information géographique : des registres « traditionnels » au développement de l'information géographique « bénévole » selon Budhathoki (2008)

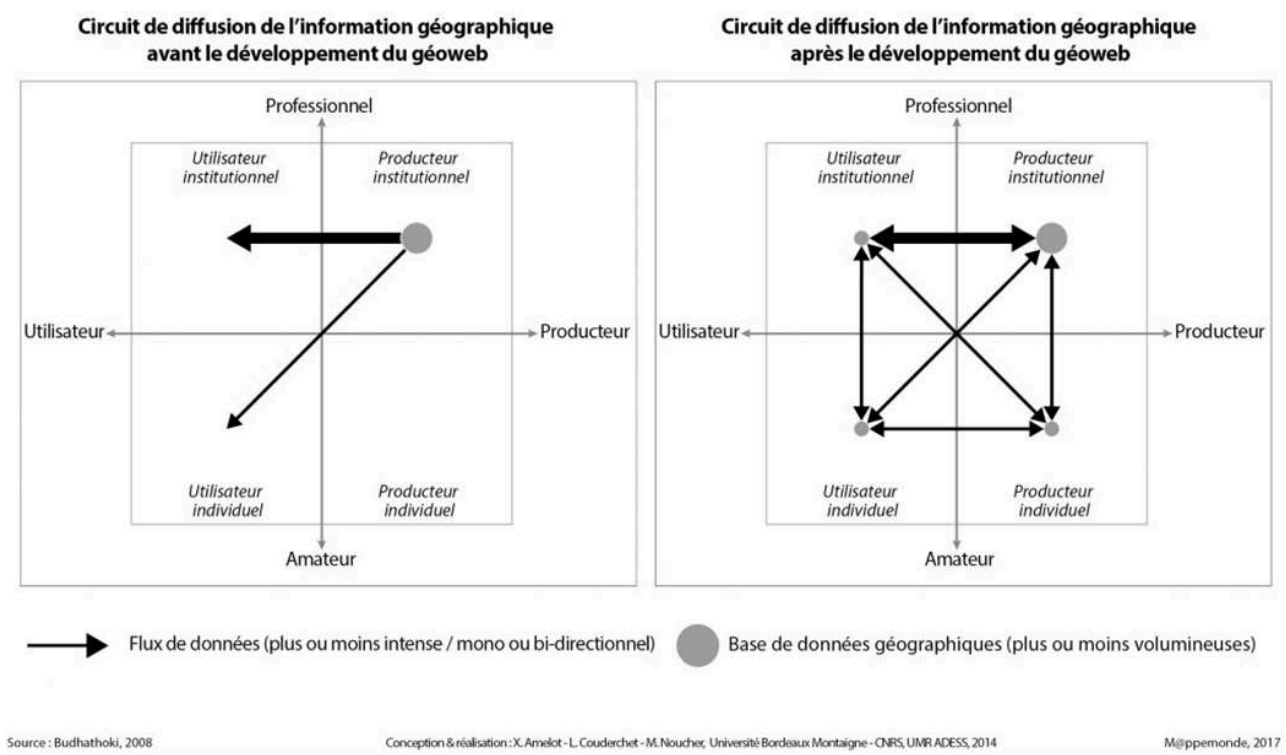

Conception et réalisation : X. Amelot - L. Couderchet - M. Noucher, Université Bordeaux Montaigne CNRS, UMR ADESS, 2014 ; source : Budhathoki, 2008

Si ce schéma a le mérite de traduire visuellement la démultiplication et la complexification des flux d'information géographique, sa structuration autour de deux couples de catégories (amateur/expert et producteur/utilisateur) est très réductrice. D'une part, la malléabilité des données géographiques numériques rend leur remobilisation aisée et estompe ainsi les limites entre producteurs et utilisateurs... devenus des «produsers» (Bruns et Schmidt, 2011); d'autre part, la massification des technologies de l'information géographique ainsi que le développement des logiciels libres et des données ouvertes permettent la circulation des compétences et le potentiel basculement de l'expertise entre professionnels et amateurs... hybridés en " proam» (Leadbeater et Miller, 2004). La base ZniefF comme les atlas de faune apparaissent, dès lors, comme deux exemples emblématiques de ce rapprochement (voire de cet efFacement) des catégories qui accompagne la complexification des flux d'information géographique (figure 6). 
Figure 6. Complexification des flux d'information géographique et effacement des catégories d'acteurs

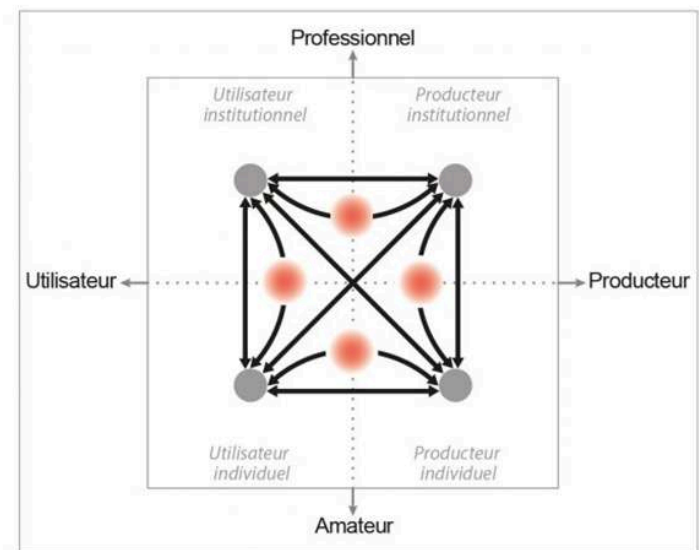

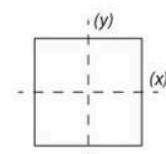

Source: Budhathoki, 2008

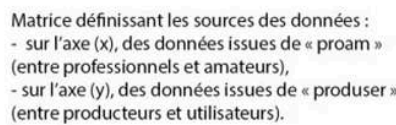
(entre producteurs et utilisateurs).

Base de données géographiques

Base de données géographiques hybrides

$\longleftrightarrow$ Flux bi-directionnel de données géographiques

Proposition d'adaptation du schéma de Budhathoki (cf. figURE 5).

Conception et réalisation : X. Amelot - L. Couderchet - M. Noucher, Université Bordeaux Montaigne CNRS, UMR ADESS, 2014 ; source : Budhathoki, 2008

\section{BIBLIOGRAPHIE}

AFIS (2015). « Sciences "participatives" » : plus de science ou détournement de la science?». Science... \& pseudo sciences, site de l'Association Française pour l'Information Scientifique, mis en ligne le 28 juillet 2015, consulté le 31 août 2015. En ligne : https://www.pseudo-sciences.org/ Sciences-participatives-plus-de-science-ou-detournement-de-la-science

AFIS (2013). « "Sciences citoyennes" : une version postmoderne de la "science prolétarienne" s'introduit au CNRS ». Science... \& pseudo sciences, site de l'Association Française pour l'Information Scientifique, mis en ligne le 13 mars 2013, consulté le 31 août 2015. En ligne : https:// www.pseudo-sciences.org/Sciences-citoyennes-une-version-postmoderne-de-la-scienceproletarienne-s

ALLAG-DHUISME F., AMSALLEM J., BARTHOD C., DESHAYES M., GRAFFIN V., LEFEUVRE C., SALLES E., BARNETCHE C., BROUARD-MASSON J., DELAUNAY A., GARNIER C.C., TROUVILIEZ J. (2010). Guide 1 : Choix stratégiques de nature à contribuer à la préservation et à la remise en bon état des continuités écologiques - premier document en appui à la mise en cuvre de la Trame verte et bleue en France. Proposition issue du comité opérationnel Trame verte et bleue. MEEDDM, consulté le 4 octobre 2011. En ligne : http:// 
www.trameverteetbleue.fr/outils-methodes/productions-comite-operationnel-trame-vertebleue

AMELOT X. (2013). « Cartographie participative pour le développement local et la gestion de l'environnement à Madagascar : Empowerment, impérialisme numérique ou illusion participative? ». L'information géographique, 2013, vol. 77, n 4, p. 47-67.

AMELOT X., COUDERCHET L. (2012). « Au-delà des ZniefF ». Les ZNIEFF, histoire d'une réussite, colloque MNHN. En ligne $:$ http://spn.mnhn.fr/colloqueZnieff2012/programme

BRUNS A., SCHMIDT J.H. (2011). « Produsage: A Closer Look at Continuing Developments ». New Review of Hypermedia and Multimedia, vol. 17, $\mathrm{n}^{\circ}$ 1, p. 3-7.

BUDHATHOKI N.R., BRUCE B., NEDOVIC-BUDIC Z. (2008). « Reconceptualizing the role of the user of spatial data infrastructure ». GeoJournal : An International Journal on Geography, vol. 72, n 3, p. 149-160. En ligne : http://www.springerlink.com/content/4tv7j5564r54u322

COUDERCHET L., AMELOT X. (2010). «Faut-il brûler les ZniefF ? ». Cybergeo : European Journal of Geography, Espace, Société, Territoire, document 498. En ligne : https://journals.openedition.org/ cybergeo/23052

DEVILLERS R., JEANSOULIN R. (2005). Qualité de l'information géographique. Paris: Hermès Lavoisier, 348 p. DODGE M., KITCHIN R. (2007). « Rethinking maps ». Progress in Human Geography, vol. 31, n 3, p. 331-344.

FLANAGIN A. J., METZGER M. J. (2008). « The credibility of volunteered geographic information ». GeoJournal, vol. 72, n 3, p. 137-148.

GOODCHILD M.F. (2007). «Citizens as sensors: The world of volunteered geography ». GeoJournal, vol. $69, \mathrm{n}^{\circ} 4$, p. 211-221.

HARLEY J. B. (1988). « Maps, knowledge and power ». In D. COSGROVE, S. DANIELS (éd.), The Iconography of Landscape. Cambridge : University of Cambridge Press, p. 277-312.

JOLIVEAU T., NOUCHER M., ROCHE S. (2013). « Cartographie 2.0, vers une approche critique d'un nouveau régime cartographique ». L'Information Géographique, vol. 77, n 4, p. 29-46.

LASCOUMES P. (2007). « Gouverner par les cartes ». Genèses, n 68, p. 2-3.

LASCOUMES P., LE GALÈS P. (2005). Gouverner par les instruments. Paris : Presses de Sciences Po, coll. «Académique », 369 p. ISBN 978-2-7246-0949-3

LAVE J., WENGER É. (1991). Situated learning: Legitimate peripheral participation. Cambridge University Press, 138 p. ISBN 9780521423748

LEADBEATER C., MILLER P. (2004). The pro-am revolution. Éd. Demos, 70 p.

LIGER J., COUZI L., FILIPPI-CODACCIONI O. (2013). « Faune Aquitaine : portail collaboratif sur la biodiversité régionale ». Netcom. Réseaux, communication et territoires, vol. 27, n 1-2, p. 224-231. En ligne : https://journals.openedition.org/netcom/1357

LIGER J. (2013). Une application de science participative : www.faune-aquitaine.org. Mémoire master 2 GTDD, LPO, UMR ADESS CNRS, Université de Bordeaux 3, 79 p.

LINARÈs S., MOURGUIART C. (2013). « BD Carthage ${ }^{\circledR}$ Guyane ». Netcom. Réseaux, communication et territoires, vol. 27, n 1-2, p. 232-236. En ligne : https://journals.openedition.org/netcom/1357 
MATHEVET R., LEPART J., MARTY P. (2013). « Du bon usage des ZNIEFF pour penser les territoires de la biodiversité ». Développement durable et territoires, vol. 4, n 1|Avril 2013. En ligne : http:// journals.openedition.org/developpementdurable

MEDTL - Ministère de l'Écologie, du développement durable, des transports et du logement (2010). Stratégie nationale pour la biodiversité 2011-2020.60p.

MERICSKAY B., ROCHE S. (2011). « Cartographie 2.0 : le grand public, producteur de contenus et de savoirs géographiques avec le web 2.0 ». Cybergeo : European Journal of Geography. En ligne :

https://journals.openedition.org/cybergeo/24710

NOUCHER M. (2017). Les petites cartes du web. Analyse critique des nouvelles fabriques cartographiques. Éditions rue d'Ulm, en version électronique et impression à la demande, 70 p.

NOUCHER M., GAUTREAU P. (2013). « Le libre accès rebat-il les cartes? Nouvelles perspectives pour les données géographique ». Les Cahiers du Numérique, vol. 9, n 1, p. 57-83.

ODER J. (2011). « Vers la structuration d'une filière aurifère "durable" ? Étude du cas de la Guyane française ». EchoGéo, n 17. En ligne : https://journals.openedition.org/echogeo/12587

PALSKY G. (2013). « Cartographie participative, cartographie indisciplinée ». L'Information Géographique, vol. 77, n 4, p. 10-25.

PICKLES J. (2004). A history of space: cartographic reason, mapping and the geo-coded world. Londres : Routledge, 233 p. ISBN 9780415144971

RAZER N. (2014). « Cartographie des services écosystémiques à partir des données de sciences participatives et citoyennes ». Mémoire master 1 GTDD, LPO, UMR ADESS CNRS, université Bordeaux Montaigne, 67 p. + annexes.

ROBINSON A.H. (1952). The look of maps. An examination of cartographic design. Madison : University of Wiconsin Press.

SIBLET J.P. (2012). « Table ronde générale ». Les ZNIEFF, histoire d'une réussite, colloque MNHN. En ligne : http://spn.mnhn.fr/colloqueZnieff2012/programme

TSAYEM DEMAZE M. (2008). « Le parc amazonien de Guyane française : un exemple du difFIcile compromis entre protection de la nature et développement ». Cybergeo : European Journal of Geography, Environnement, Nature, Paysage, document 416. En ligne : https:// journals.openedition.org/etudescaribeennes/315

TRANSLER A.-L., SAFFACHE P., MOULLET D. (2006). « L'activité aurifère en Guyane : contexte et perspectives ». Études caribéennes, $n^{\circ}$ 5. En ligne : https://journals.openedition.org/ etudescaribeennes/315

\section{NOTES}

1. Souvent traduite par "information géographique volontaire », VGI a toutefois un sens plus proche d'information géographique bénévole, comme le rappelle Gilles Palsky (2013).

2. L'appel à communication du colloque sur le thème «Fronts et frontières des sciences du territoire » est disponible à l'adresse suivante : http://cist2014.sciencesconf.org/resource/page/ $\mathrm{id} / 6$

3. Zone naturelle d'intérêt écologique, faunistique et floristique. Écrire ce sigle en minuscules permet d'insister sur le fait qu'il s'agit d'un acronyme (il peut se lire et se prononcer) en voie de lexicalisation. 
4. Dans le sens du crowd de crowdsourcing.

5. Ces critères sont définis dans la norme ISO 19113 à l'aide de méthodes explicitées dans la norme ISO 19114 et de mesures spécifiques dans la norme 19138. Pour communiquer cette évaluation de qualité on utilise les métadonnées de la norme ISO 19115.

6. Les anglophones parlent de «fitness for use ».

7. Expression reprise de la loi de protection des sites de 1906. Son article premier fixe les principes de constitution de la commission, dont les membres sont « choisis parmi les notabilités des arts, des sciences et de la littérature ".

8. À titre d'exemple, alors qu'en 1994, les agences de l'eau et l'IGN créent, suite à une circulaire de 1991 (n 91-50 du 12 février 1991), la BD CARTHAGE ${ }^{\oplus}$ (Base de Données sur la CARtographie THématique des AGences de l'eau et du ministère chargé de l'environnement), il faudra attendre près de 20 ans pour que soit diffusé un référentiel hydrographique qui réponde aux spécificités du territoire guyanais (Linarès et Mouguiard, 2013).

9. La population guyanaise est concentrée à $90 \%$ sur la bande littorale. Les sièges des principales associations de protection de l'environnement, des bureaux d'étude et des services de l'État sont situés pour l'essentiel dans trois quartiers (Buzaret, Montabo et Bourda) du nord de Cayenne.

10. L'éloignement de la Guyane conduit, comme c'est le cas de la plupart des territoires ultramarins, à un très fort turn over d'agents en provenance de métropole. Cependant, un noyau d'acteurs fortement ancré sur le territoire est présent depuis plusieurs années en occupant des postes variés. Ainsi, nous avons pu rencontrer, un agent de l'État (DEAL), membre du GEPOG et travaillant précédemment dans un bureau d'étude ou un ancien agent de l'Office National des Forêts (ONF) travaillant pour le Parc Amazonien de Guyane et impliqué avec des scientifiques sur un programme de cartographie participative avec les communautés amérindiennes Teko et Wayãpi.

\section{RÉSUMÉS}

Les politiques de conservation de la biodiversité, grandes consommatrices d'informations géographiques, sont aujourd'hui confrontées à un double mouvement: la faiblesse des moyens consacrés par les pouvoirs publics à la reconnaissance des milieux et la montée en puissance des initiatives contributives. La connaissance ofFIcielle qui peine à progresser est ainsi débordée par les démultiplications d'informations réunies et diffusées en dehors des circuits officiels par l'intermédiaire d'Internet. Nous discutons la légitimité de ces données et leur recevabilité par les politiques de conservation.

Conservation policies for biodiversity, major consumers of spatial data, is now facing a double movement: the lack of public funding for environmental recognition and the rise of participatory mapping. OfFIcial information, which is developing with difficulty, is today overwhelmed by the plethora of information unofficially gathered and published on the Internet. This is a discussion of the legitimacy of such data and its admissibility by conservation policies.

Las políticas para la conservación de la biodiversidad necesitan disponer de gran cantidad de información geográfica. Actualmente existe una gran controversia : hay una escasez de recursos públicos para procurar estos datos, pero aumenta exponencialmente la disponibilidad de fuentes e información colaborativa. Esta parálisis del sector oficial contrasta con la abrumadora oferta de 
información accesible en Internet desligada del sector público, por lo que es importante valorar la calidad y validez de la misma para las políticas de conservación.

\section{INDEX}

Thèmes : Enjeux de l'information territoriale locale ouverte et participative

Keywords : web-mapping 2.0, volunteered geographic information, data legitimacy, biodiversity politics

Mots-clés : cartographie contributive, information géographique bénévole, légitimité des données, politiques de biodiversité

Palabras claves : Cartografía colaborativa, información geográfica voluntaria, legitimidad de la información, políticas de biodiversidad

\section{AUTEURS}

\section{XAVIER AMELOT}

Maître de conférence, Université Bordeaux Montaigne, UMR ADESS

LAURENT COUDERCHET

Professeur, Université Bordeaux Montaigne, UMR ADESS

\section{MATTHIEU NOUCHER}

CNRS, UMR Passages, Bordeaux 